\title{
Anticancer activity of sugiol against ovarian cancer cell line SKOV3 involves mitochondrial apoptosis, cell cycle arrest and blocking of the RAF/MEK/ERK signalling pathway
}

\author{
Yan Wang ${ }^{1}$, Liang-Yan $\mathrm{Shi}^{1}$, Wei-Hong $\mathrm{Qi}^{2}$, Jing Yang ${ }^{3}$, Yue $\mathrm{Qi}{ }^{4}$
}

\author{
${ }^{1}$ Department of Gynaecology, Hubei Maternity and Child Health Hospital, Wuhan, \\ China \\ 2Department of Obstetrics and Gynaecology, Beijing Hospital, Beijing, China \\ ${ }^{3}$ Department of Obstetrics and Gynaecology, Haidian Woman's and Children's \\ Hospital, Beijing, China \\ ${ }^{4}$ Department of Obstetrics and Gynaecology, Dongguan Hospital of Traditional \\ Chinese Medicine, Dongguan Guangdong, China
}

Submitted: 21 April 2017

Accepted: 9 September 2017

Arch Med Sci 2020; 16 (2): 428-435

DOI: https://doi.org/10.5114/aoms.2017.71420

Copyright @ 2017 Termedia \& Banach

\section{Abstract}

Introduction: Ovarian cancer is one of the leading causes of cancer-related deaths in women. Treatments for ovarian cancer include surgery followed by chemotherapy. However, the survival rate for ovarian cancer is still not satisfactory. Moreover, the current chemotherapy has numerous associated side effects. Therefore there is an urgent need to look for novel and more viable treatment options. Against this backdrop the present study was designed to evaluate the anticancer activity of sugiol against ovarian cancer cells.

Material and methods: Cell viability was assessed by CCK8 assay, apoptosis by DAPI, AO/ER and annexin V/PI staining. Mitochondrial membrane potential and cell cycle analysis was performed by flow cytometry. Cell migration was investigated by wound healing assay. Protein expression was monitored by western blotting.

Results: The results of the present study indicated that sugiol exerts significant $(p<0.0001)$ anticancer effects on SKOV3 cancer cells with an IC $C_{50}$ of $25 \mu \mathrm{M}$. However, sugiol exhibited less cytotoxicity against normal ovarian cells with an $\mathrm{IC}_{50}$ of $62.5 \mu \mathrm{M}$. The anticancer effects of sugiol were found to be due to GO/G1 cell cycle arrest and mitochondrial apoptosis. Sugiol also inhibited cell migration of SKOV3 cells dose dependently. Moreover, the results showed that sugiol could inhibit the RAF/MEK/ERK signalling pathway in a dose-dependent manner.

Conclusions: The results of the present study indicate that sugiol exerts potent anticancer effects on SKOV3 cells via induction of cell cycle arrest, mitochondrial apoptosis and inhibition of the RAF/MEK/ERK signalling pathway.

Key words: ovarian cancer, cell cycle arrest, apoptosis, RAF/MEK/ERK, cell migration.

\section{Introduction}

Ovarian cancer is a frequently detected cancer in women, and about $90 \%$ of ovarian cancers are epithelial ovarian cancer (EOC). Epithelial ovarian cancer is a very malignant cancer in the female reproductive

\author{
Corresponding author: \\ Wei-Hong Qi PhD \\ Department of Obstetrics \\ and Gynaecology \\ Beijing Hospital \\ 1 Dongdan Dahua Road \\ 100730 Beijing, China \\ Phone: +861085136112 \\ E-mail:WCaroleengon@ \\ yahoo.com
}


system and is associated with very high morbidity and mortality rates [1]. Most of these cancers are diagnosed at very advanced stages, and treatment at these stages becomes almost impossible. The 5 -year survival rate is only $30 \%$ for EOC. The main treatment options for EOC include surgery followed by chemotherapy [2]. However, early metastasis, late diagnosis, limited treatment options, drug resistance and the side effects associated with the available drugs are some of the major concerns in the treatment of EOCs. Consistent with these issues, there is a pressing need to explore more efficient, effective and viable treatment options for EOC.

Natural products have long been considered to be important sources of anticancer drugs [3]. Epidemiological studies have revealed that high dietary intake of naturally occurring plant secondary metabolites, such as flavonoids, through vegetables, fruits and beverages, are associated with lower risks of cancer. This is further justified by several in vitro and in vivo studies [4]. Nonetheless, it has been reported that out of the 155 small-molecule anticancer drugs, the number of drugs that are natural products and/or synthesized from natural products accounts for $72.9 \%$ [5]. Among natural sources, plants represent an important source of anticancer drugs; and several plant-derived compounds are currently used as anticancer drugs or are being assessed in clinical trials [6-8].

Sugiol is a diterpene and has been isolated from several species of the genus Salvia [9, 10]. Previously it has been reported that sugiol exhibits anticancer activity against a range of cancer types such as colon, breast and lung cancer [11]. Therefore, in the present study we determined the anticancer activity of sugiol against breast cancer cells. The mechanism for the anticancer activity of sugiol has not been explicitly explored and RAF/mitogen activated protein (MAP)/extracellular signal-regulated kinase (ERK) (MEK)/ERK (RAF/MEK/ERK) signalling pathways have been reported to play important roles in the tumourigenesis and progression of several cancers including ovarian cancer [12]. Therefore, we also investigated the effect of sugiol on the RAF/MEK/ERK signalling pathway.

\section{Material and methods}

\section{Chemicals, reagents and cell cultures}

Sugiol and other chemicals were of reagent grade and purchased from Sigma Chemical Co. (St. Louis, Missouri, USA) unless otherwise mentioned. The normal human ovarian epithelial cell line (Moody) and the human ovarian tumour cell line (SKOV3) were obtained from Type Culture Collection of Chinese Academy of Scienc- es, Shanghai, China. The cells were cultured in RPMI-1640 medium containing 10\% fetal bovine serum, $100 \mathrm{U} / \mathrm{ml}$ penicillin and $100 \mu \mathrm{g} / \mathrm{ml}$ streptomycin and maintained in a humidified atmosphere containing $5 \% \mathrm{CO}_{2}$.

\section{CCK8 assay for assessment of cell viability}

The cell viability of the normal ovarian cells (Moody) and the ovarian cancer cells (SKOV3) was determined by CCK 8 assy. In brief, $5 \times 10^{3}$ cells were seeded on a 96-well plate and then kept at $37^{\circ} \mathrm{C}$ for incubation in a humidified, $5 \% \mathrm{CO}_{2}$ atmosphere. After overnight incubation the cells were treated with different concentrations of sugiol $(0-200 \mu \mathrm{M})$ for $24 \mathrm{~h}$. Then, $10 \mu \mathrm{l}$ of CCK8 was added to each well and again incubated at $37^{\circ} \mathrm{C}$ for $1 \mathrm{~h}$. The optical density at $\mathrm{OD}_{450} \mathrm{~nm}$ was determined by a microplate spectrophotometer (BioRad, Segrate, Italy). The cell viability was presented as percentage of the control.

\section{AO/EB, DAPI, annexin V/PI staining}

SKOV3 cells were seeded at the density of $2 \times 10^{5}$ cells/well in 6-well plates and administered with different concentrations of Sugiol for $24 \mathrm{~h}$. The cells were then stained with a mixture of $A O$ and EB. Stained cells were examined under the fluorescent microscope. DAPI staining was carried by incubating the cells in 6-well plates for 20 min in the dark and treated with different concentrations of sugiol. The cells were then washed with PBS, fixed in formaldehyde (10\%) and then again washed with PBS. The DAPI stained cells were then examined by fluorescence microscope. For annexin V/PI a similar procedure as that of DAPI was followed except the cells were stained with annexin V/PI and investigated by flow cytometry.

\section{Determination of mitochondrial membrane potential (MMP)}

SKOV3 cells were seeded at a density of $2 \times 10^{5}$ cells/well in a 6-well plate and incubated for $24 \mathrm{~h}$ and treated with $0,12.5,25$ and $50 \mu \mathrm{M}$ sugiol for $48 \mathrm{~h}$ at $37^{\circ} \mathrm{C}$ in $5 \% \mathrm{CO}_{2}$ and $95 \%$ air. Then cells from all samples were collected, washed twice with PBS and re-suspended in $500 \mu \mathrm{l}$ of DiOC6 $(1 \mu \mathrm{mol} / \mathrm{l})$ for $\mathrm{MMP}$ at $37^{\circ} \mathrm{C}$ in a dark room for $30 \mathrm{~min}$. The samples were then examined instantly using a flow cytometer as described previously in the literature.

\section{Cell cycle analysis}

The cell cycle analysis was carried out by flow cytometry after staining with propidium iodide. Briefly, SKOV3 cells were plated at the density of $2 \times 10^{5}$ and administered with different concen- 
trations of sugiol $(0,12.5,25$ and $50 \mu \mathrm{M})$ and the cells were harvested and kept in ethanol (70\%) at $4^{\circ} \mathrm{C}$. After overnight incubation the cells were collected again by centrifugation at $1000 \mathrm{rpm}$ for 10 min and washed with PBS and finally suspended in $250 \mathrm{ml}$ of PBS and treated with RNase for 20 min. Afterwards the cells were stained with propidium iodide and the cell cycle analysis was carried out by flow cytometry.

\section{Cell migration assay}

Cell migration of SKOV3 cells was determined by wound healing assay. Briefly, cells were cultured until confluence. Then the SKOV3 wells were treated with $0,12.5,25$ and $50 \mu \mathrm{M}$ (concentrations lower than $\mathrm{IC}_{50}$ because doses higher than $\mathrm{IC}_{50}$ exhibit considerable cytotoxic effects) of sugiol and the cells were scratched with a sterile pipette tip and then incubated for $24 \mathrm{~h}$. The wound healing capacity of sugiol-treated cells was determined by comparing the wound length with that of the untreated control cells.

\section{Protein expression by western blotting}

Total protein from untreated and sugiol-treated cells was isolated in RIPA lysis buffer. Equal protein extracts from each group were run on SDS PAGE and then transferred to a polyvinylidene fluoride membrane. This was followed by blocking with $5 \%$ non-fat milk and incubation at room temperature for $1 \mathrm{~h}$. Then the membranes were incubated with a specific primary antibody at $4^{\circ} \mathrm{C}$ overnight. This was followed by washing in washing buffer and incubation for $1 \mathrm{~h}$ with a suitable secondary antibody. The protein bands of interest were visualised by an ECL Advanced Western Blot Detection Kit (GE Healthcare, Uppsala, Sweden).

\section{Statistical analysis}

Data were expressed as mean of the replicates $\pm \mathrm{SD}$. Statistical significance and $\mathrm{IC}_{50}$ values were analyzed by GraphPad Prism Demo, Version 5 (GraphPad Software, San Diego, California, USA). ${ }^{\star} P<0.05,{ }^{* *} p<0.01,{ }^{* * *} p<0.001$ were considered as indicating a significant difference.

\section{Results}

Sugiol exerts antiproliferative effects on SKOV3 cells

The antiproliferative effect of sugiol against human ovarian SKOV3 cancer cells and human normal Moody ovarian cells was evaluated by CCK-8 assay. The results indicated that sugiol exerted dose-dependent antiproliferative effects on SKOV3. However, sugiol was found to be less cytotoxic against the normal cells (Figure $1 \mathrm{~A}$ ). The $\mathrm{IC}_{50}$ of sugiol against SKOV3 cells was found to be $25 \mu \mathrm{M}$ as compared to the $\mathrm{IC}_{50}$ of $62.5 \mu \mathrm{M}$ against the normal ovarian cells. Moreover, sugiol also influenced the cell morphology of SKOV3 cells (Figure 1 B). As the concentration of sugiol was increased in the SKOV3 cancer cells they turned round, contracted and separated from the substratum (Figure 1 B), which are the chief morphological alternations associated with apoptosis.

\section{Sugiol triggers mitochondrial apoptosis}

Since sugiol induced morphological changes in SKOV3 cells that are characteristic of apoptosis, we carried out DAPI and AO/EB staining. The results indicated that sugiol induced apoptosis in SKOV3 cells, as evident from the increasing number of white nuclei in the case of DAPI staining (Figure $2 \mathrm{~A}$ ) and orange fluorescence in the
A

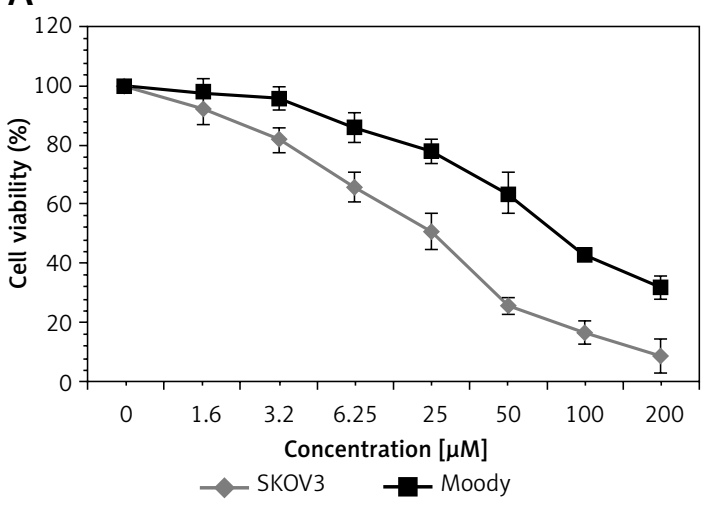

B



Figure 1. Assessment of cell viability by CCK8 assay $(n=9)(\mathbf{A})$ and effect on cell morphology (B). All results were carried out three times in triplicate $(n=9)$ and the results were expressed as mean \pm SD 
A

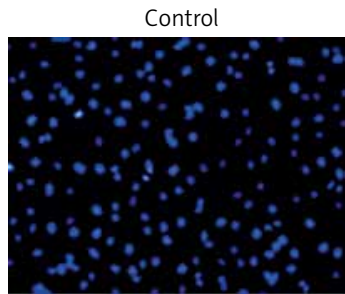

$25 \mu \mathrm{M}$

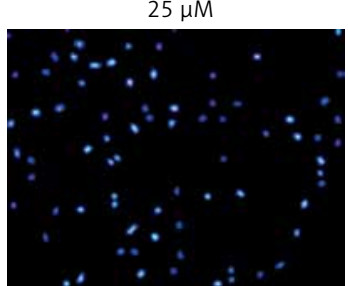

B



$50 \mu M$

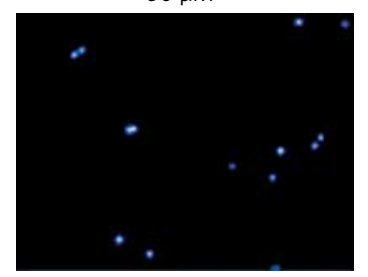

Control

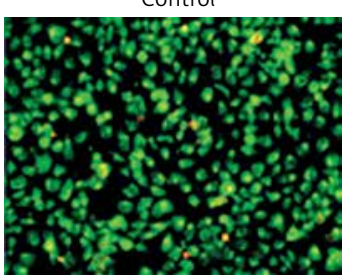

$25 \mu \mathrm{M}$

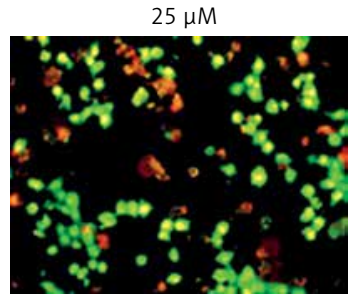

$12.5 \mu \mathrm{M}$



$50 \mu \mathrm{M}$

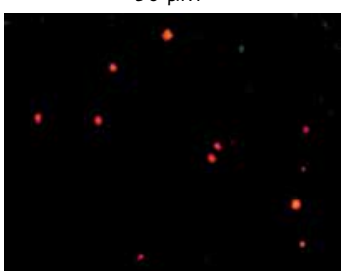

Figure 2. Sugiol induces apoptosis in SKOV3 cells as depicted by DAPI staining (A), AO/EB staining (B). The experiments were carried out in triplicate

case AO/EB staining (Figure 2 B). To estimate the apoptotic cell populations, we carried out annexin V/IP staining, and it was observed that apoptotic cells increased with increase in the concentration of sugiol (Figure 3). The apoptotic cell populations were found to be 5.6, 18.8, 29.7 and $61.3 \%$ at the concentrations of $0,12.5,25$ and $50 \mu \mathrm{M}$ of sugiol. Next, we determined the effect of different concentrations of sugiol on MMP. The results indicated that sugiol treatment decreased the MMP of SKOV3 cells in a dose-dependent manner (Figure $4 \mathrm{~A}$ ). The inhibition of cell migration was also associated with the concomitant upregulation of Bax and downregulation of $\mathrm{BCl}-2$ expression (Figure 4 B).

\section{Sugiol induces G0/G1 cell cycle arrest}

Cell cycle arrest is an important mechanism by which anticancer agents exert their inhibitory effects. Therefore we also determined the effect of sugiol on cell cycle phase distribution of sugi- ol-treated SKOV3 cells (Figures 5 A, B). Our results indicated that the percentage of SKOV3 cells was considerably increased in G0 at the concentrations of 0 to $50 \mu \mathrm{M}$ of sugiol, causing G0/G1 cell cycle phase arrest. Additionally the populations of SKOV 3 cells in G0 phase were marginally increased at a dose of $12.5 \mu \mathrm{M}$, reasonably increased at $25 \mu \mathrm{M}$, and dramatically increased at $50 \mu \mathrm{M}$, indicating a dose-dependent effect of sugiol.

\section{Sugiol inhibits cell migration}

We also examined whether sugiol could inhibit the migration of SKOV3 cancer cells at different concentrations by wound healing assay. The results of wound healing assay showed that sugiol reduced the migratory capability of SKOV3 cells in a dose-dependent manner. While as in control the cells showed fairly good capacity to migrate, in treatment, the cells showed less potential to migrate, as depicted in Figures $6 \mathrm{~A}$ and B. Additionally, we observed that sugiol caused a reduc-
A

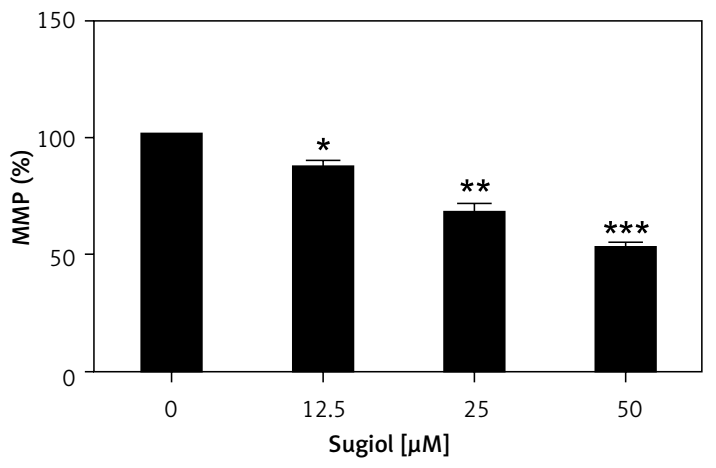

B

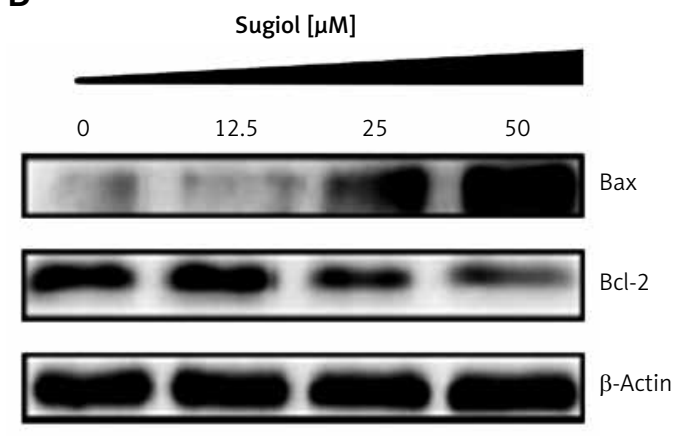

Figure 3. Effect of indicated concentrations of sugiol on (A) mitochondrial membrane potential (MMP) as determined by flow cytometry $(n=9)$, (B) expression of Bax and Bcl-2 by western blotting

All experiments were carried out three times in triplicate $(n=9)$ and expressed as mean $\pm S D$. The results were considered significant at ${ }^{*} p<0.01,{ }^{* *} p<0.001$ and ${ }^{* * *} p<0.0001$. 
A

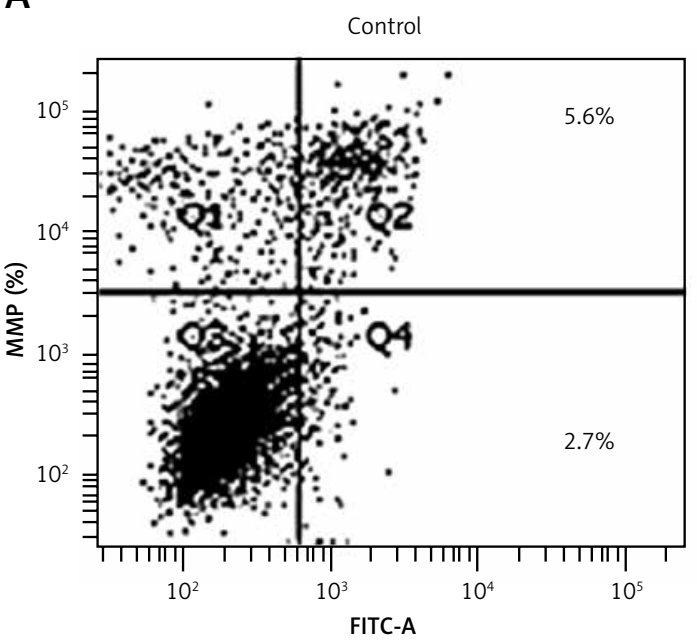

C

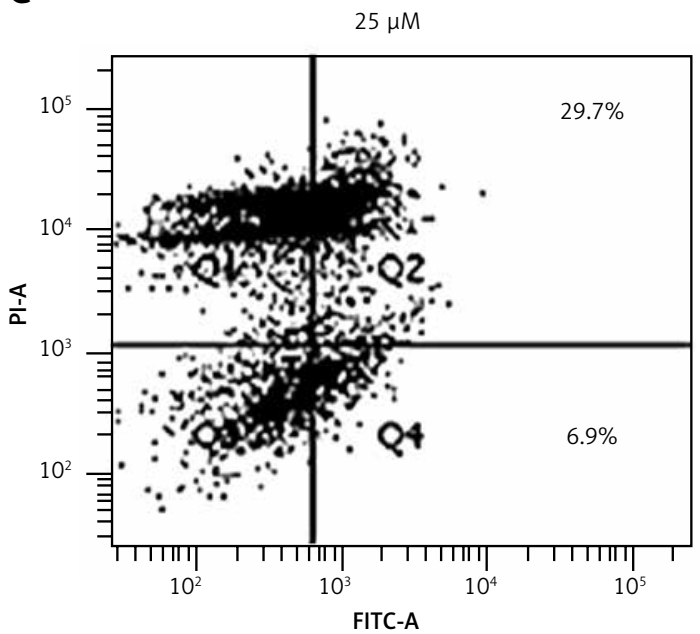

B

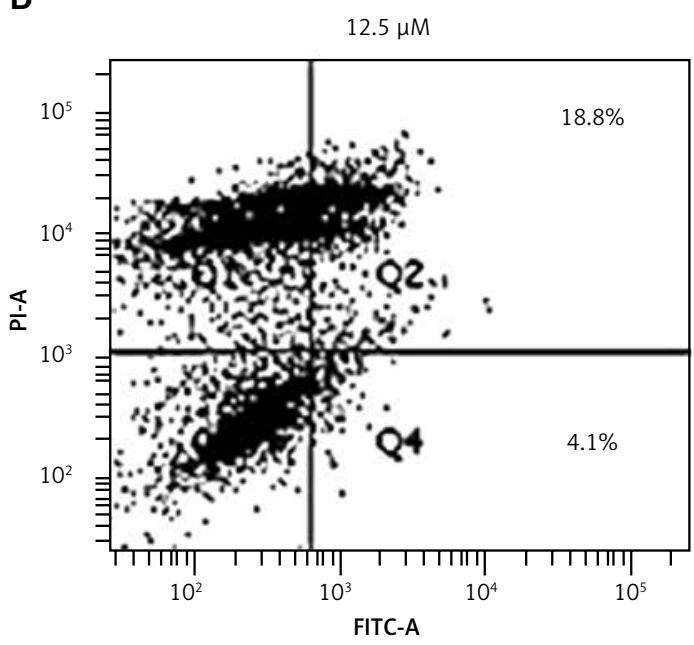

D

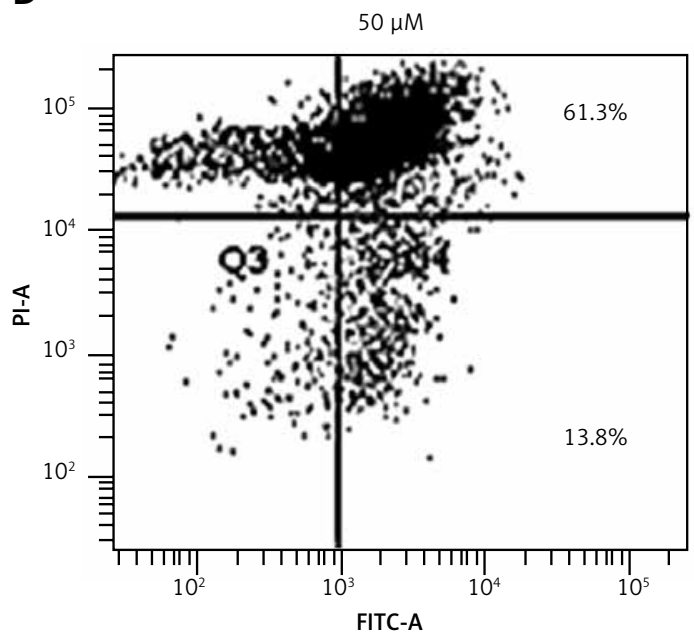

Figure 4. Estimation of apoptotic cell populations by annexin V/PI staining followed by flow cytometry. The experiment was carried out three times

tion in the expression of MMP-9 and MMP-2 in a dose-dependent manner (Figure 6 B).

\section{Sugiol blocks RAF/MEK/ERK signalling pathway}

The RAF/MEK/ERK signalling pathway has been reported to play important roles in the tumourigenesis and progression of several cancers including ovarian cancer. Therefore, we evaluated the effect of varied concentrations of sugiol on RAF/ MEK/ERK cascade. The results indicated that sugiol inhibited the phosphorylation of MEK and ERK in a dose-dependent manner (Figure 7).

\section{Discussion}

Ovarian cancer is one of the lethal cancers detected in women. It is often referred to as the silent killer as it remains symptomless and undetected until the advanced stage, at which it becomes almost impossible to treat [13]. Besides late diagnosis, poor availability of suitable drugs, rapid metastasis, development of drug resistance and toxicities associated with the chemotherapy constitute the main hurdles in the treatment of ovarian cancers [14] Therefore there is an imperative need to explore new and viable drug options. Sugiol is a diterpene and has been reported to possess tremendous pharmacological potential [11]. In the present study we evaluated the anticancer effects of sugiol against ovarian cancer cells. The results indicated that sugiol significantly inhibited the growth of SKOV3 cells $\left(\mathrm{IC}_{50} ; 25 \mu \mathrm{M}\right)$. However, sugiol showed less cytotoxicity towards normal ovarian cells $\left(\mathrm{IC}_{50} ; 62.5 \mu \mathrm{M}\right)$, indicating that sugiol targets cancer cells more potently. Our results are in agreement with previous studies in which sugiol was reported to inhibit the growth of several types of cancers including prostate cancer [11, 15-17]. Moreover, sugiol treatment triggered several morphological changes in SKOV3 cells that are characteristic of apoptosis. Taking cue from this, we carried out DAPI and AO/EB staining to examine whether sugiol induces apoptosis in SKOV3 cells. Interestingly, we observed that sugiol 
A
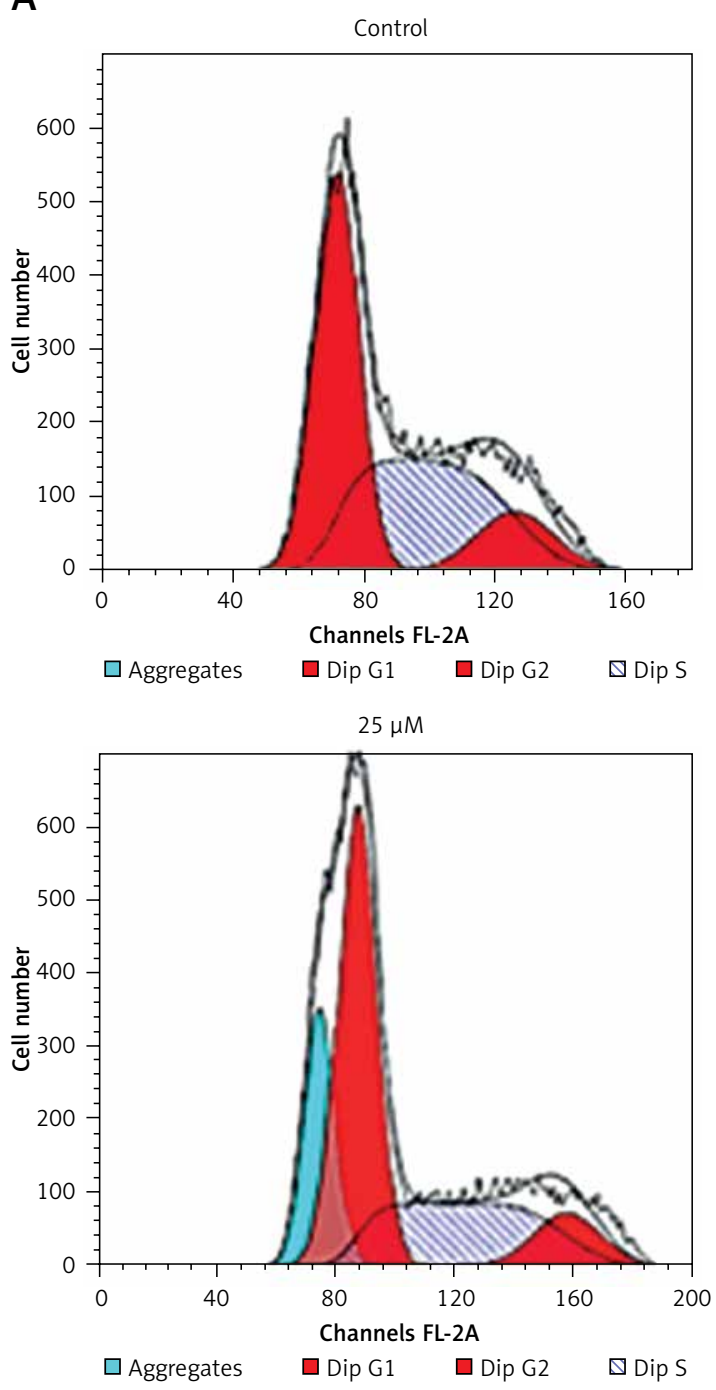

B

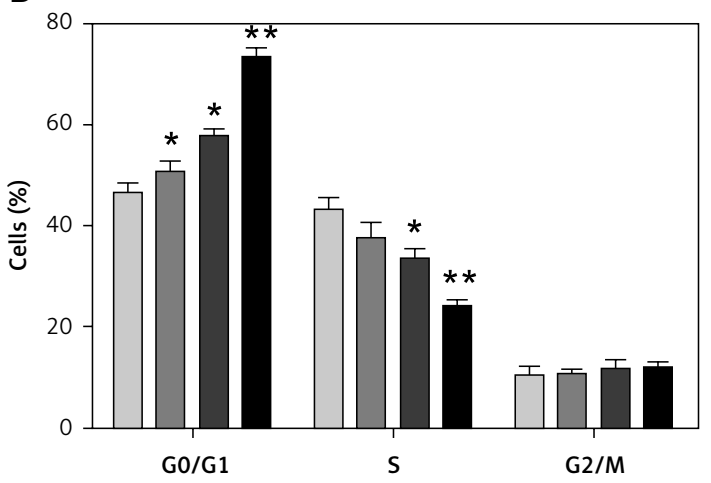

increased for the DNA fragmentation as observed by DAPI and led to an increase in the orange fluorescence as observed by AO/EB staining indicative of apoptosis. These results were further substantiated by the results of annexin V/IP staining which showed that sugiol caused a significant increase in the apoptotic cell populations of SKOV3 cells. Our results are further supported by previous studies in
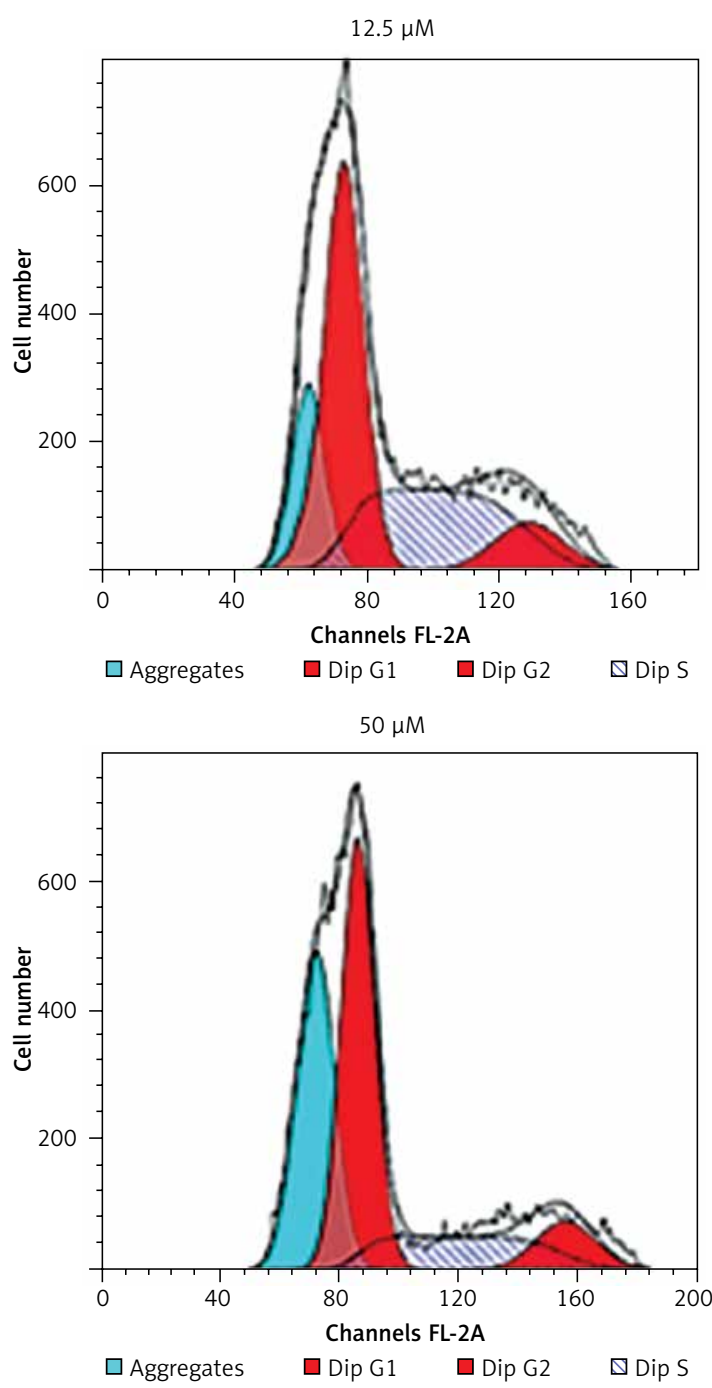

Figure 5. Effect of indicated concentrations of sugiol on cell cycle phase distribution by flow cytometry (A), estimation of cells in each phase (B)

The experiments were carried out three times in triplicate $(n=9)$. The results were considered significant at ${ }^{*} p<0.01,{ }^{* *} p<0.001$ and ${ }^{* *} p<0.0001$.

which sugiol was reported to induce apoptosis in cancer cells [18]. To determine whether the apoptosis follows the mitochondrial route we determined the effect of sugiol on MMP, and it was observed that sugiol significantly decreased the mitochondrial membrane potential, which was associated with an increase in the Bax/Bcl-2 ratio. It has been reported that drugs with apoptosis-inducing proper- 
A

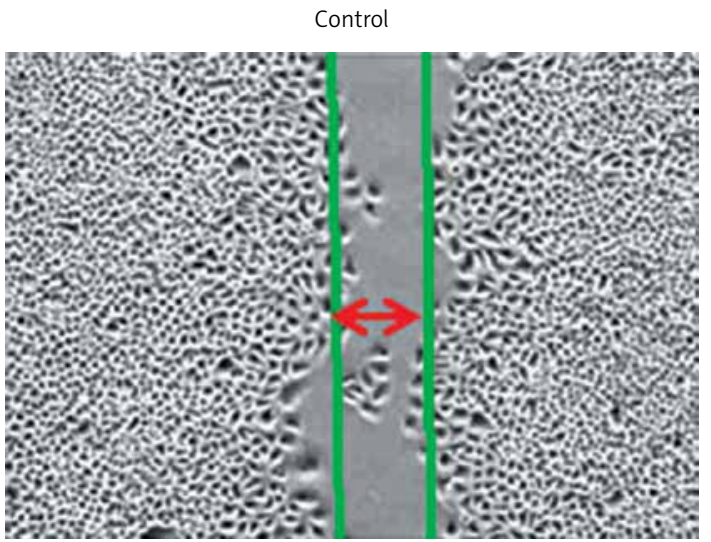

$25 \mu \mathrm{M}$

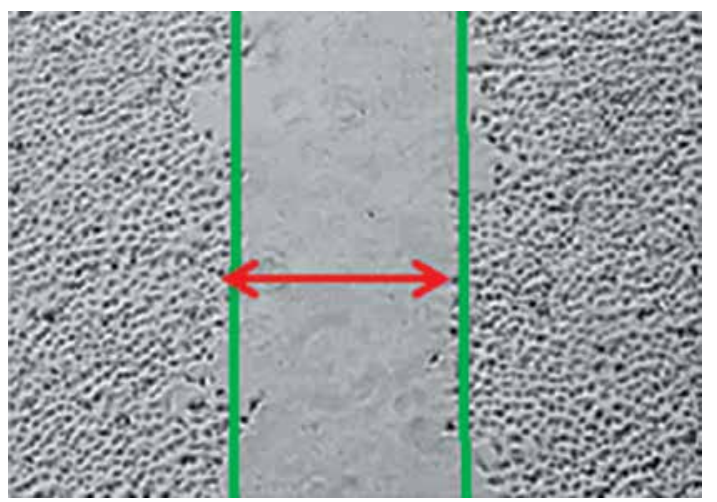

B

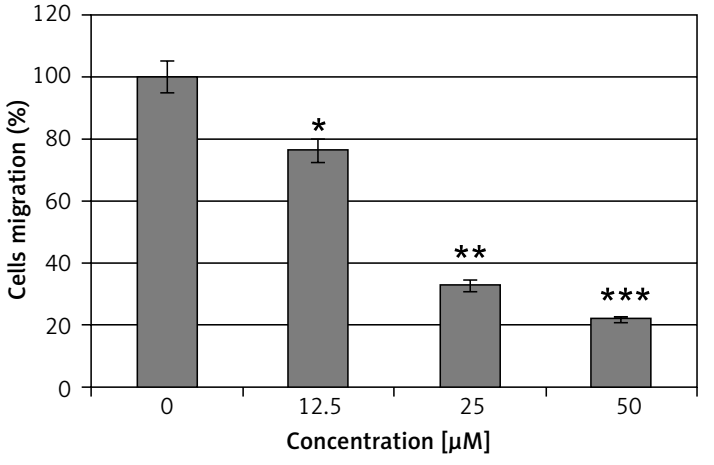

$12.5 \mu \mathrm{M}$

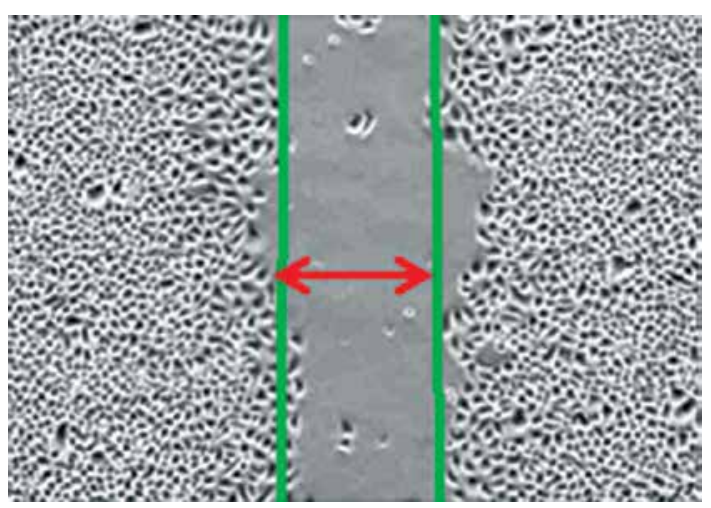

$50 \mu \mathrm{M}$

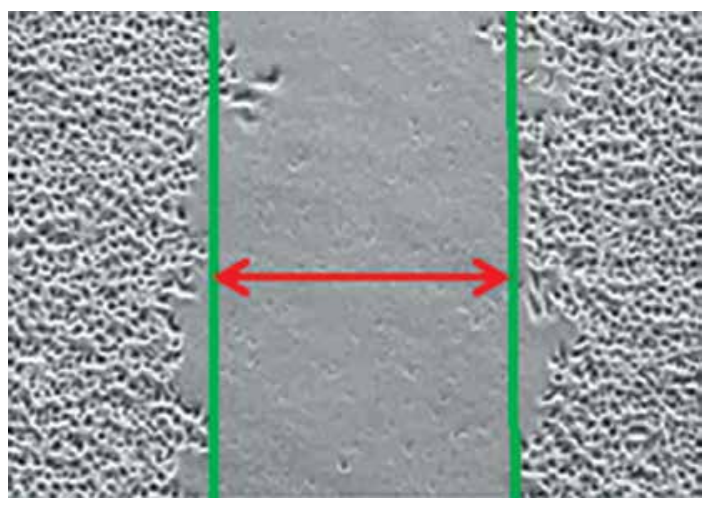

C

Sugiol $[\mu \mathrm{M}]$

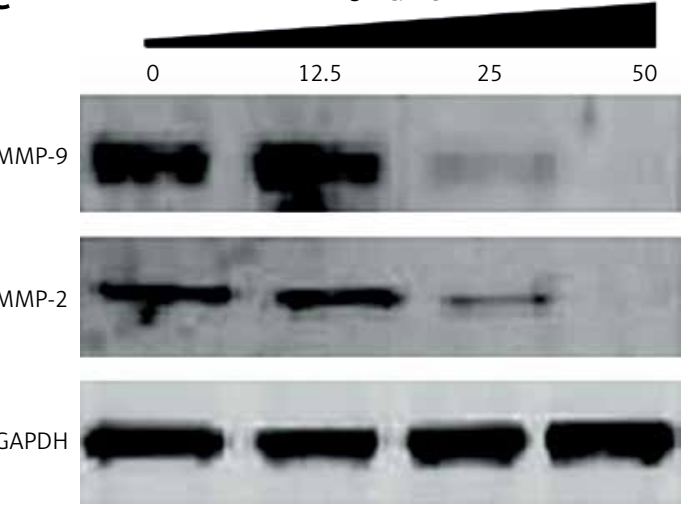

Figure 6. Effect of indicated concentrations of sugiol on cell migrations (A) wound healing assay, (B) estimation of $\%$ cell migration, (C) expression of MMP-9 and MMP-2

The experiments were carried out three times in triplicate $(n=9)$. The results were considered significant at ${ }^{*} p<0.01,{ }^{* *} p<0.001$ and ${ }^{* * *} p<0.0001$.

ties exhibit the potential to minimize potential drug resistance [19], and our results clearly indicated that sugiol has apoptosis-inducting properties, suggesting that sugiol may prove an important lead molecule for the treatment of ovarian cancer. Besides apoptosis, cell cycle arrest is another mechanism by which anticancer agents exert their effects. Our results revealed that sugiol caused G0/G1 cell cycle arrest. Apoptotic cell death is triggered when explicit checkpoints are arrested during cell division [20]. Consistent with this, several anticancer agents cause cell cycle arrest and have been found to be clinically effective for cancer treatment [20]. Cell migration is the key feature of cancer progression and metastasis [21, 22] and suppression of cell migration may prove essential in inhibition of metastasis in vivo. This may ensure longer survival of patients. In our study we observed that sugiol inhibited the migration of SKOV3 cells, which was associated with a concomitant decrease in the expression of MM-2/9. Mitogen-activated protein kinase (MAPK) cascades are key signalling pathways involved in 


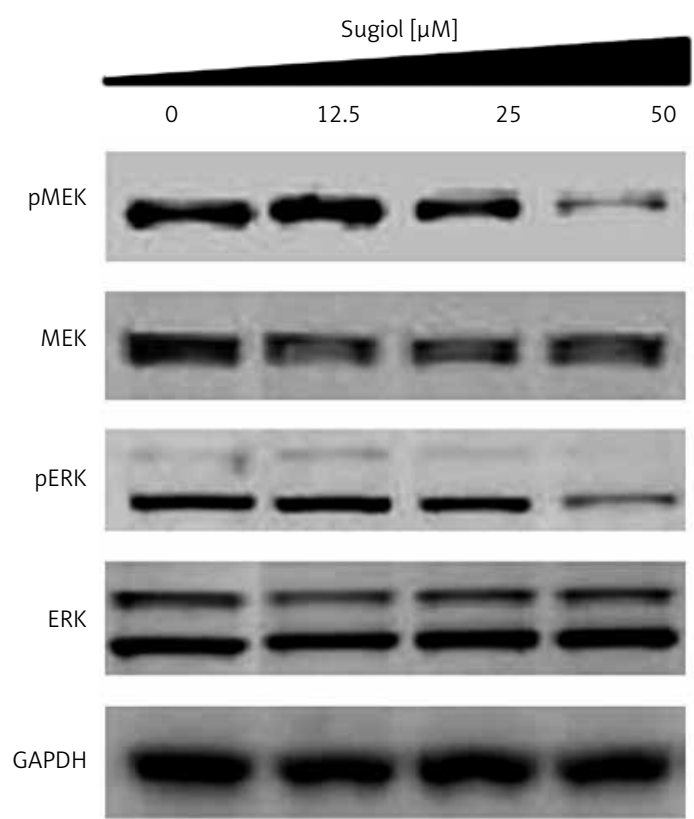

Figure 7. Effect of indicated doses of sugiol on expression of $p$-MEK and $p$-ERK by western blotting. All experiments were carried out three times in triplicate

the regulation of normal cell proliferation, survival and differentiation. Aberrant regulation of MAPK cascades contributes to cancer and other human diseases [23]. Therefore, we determined the effect of sugiol on the RAF/MEK/ERK pathway, and it was observed that sugiol could inhibit the phosphorylation of MEK and ERK, indicating that sugiol may prove a potential candidate to target this pathway. These results indicate that sugiol has potent anticancer activity. However, evaluation of sugiol against more cell lines and under in vivo conditions will enable better understanding of sugiol-induced anti-proliferative effects on ovarian cancer.

In conclusion, sugiol exerts potent anticancer effects on SKOV3 cells. These anticancer effects are attributed to the cell cycle arrest, apoptosis and inhibition of the RAF/MEK/ERK pathway. Moreover, this study opens the way for further evaluation of sugiol under in vivo conditions.

\section{Conflict of interest}

The authors declare no conflict of interest.

\section{References}

1. Gupta N, Xu Z, El-Sehemy A, Steed H, Fu Y. Notch3 induces epithelial-mesenchymal transition and attenuates carboplatin-induced apoptosis in ovarian cancer cells. Gynecol Oncol 2013; 130: 200-6.

2. Siegel R, DeSantis C, Virgo K, et al. Cancer treatment and survivorship statistics, 2012. CA: Cancer J Clin 2012; 62: 220-41.

3. Xu G, Kan WL, Zhou Y, et al. Cytotoxic acylphloroglucinol derivatives from the twigs of Garcinia cowa. J Natural Products 2010; 73: 104-8.
4. Knekt P, Kumpulainen J, Jarvinen R, et al. Flavonoid intake and risk of chronic diseases. Am J Clin Nutr 2002; 76: 560-8.

5. Newman DJ, Cragg GM. Natural products as sources of new drugs over the 30 years from 1981 to 2010. J Nat Prod 2012; 75: 311-35.

6. Cragg GM, Grothaus PG, Newman DJ. Impact of natural products on developing new anti-cancer agents. Chem Rev 2009; 109: 3012-43.

7. Shah U, Shah R, Acharya S, Acharya N. Novel anticancer agents from plant sources. Chin J Nat Med 2013; 11: 16-23.

8. Zhang X, Chen LX, Ouyang L, Cheng Y, Liu B. Plant natural compounds: targeting pathways of autophagy as anti-cancer therapeutic agents. Cell Prolif 2012; 45: 466-76.

9. Liu J, Zapp J, Becker H. Comparative phytochemical investigation of Salvia miltiorrhiza and Salvia triloba. Planta Med 1995; 61: 453-5.

10. Ulubelen A, Öksüz S, Kolak U, Bozok-Johansson C, Çelik C, Voelter W. Antibacterial diterpenes from the roots of Salvia viridis. Planta Med 2000; 66: 458-62.

11. Jung SN, Shin DS, Kim HN, et al. Sugiol inhibits STAT3 activity via regulation of transketolase and ROS-mediated ERK activation in DU145 prostate carcinoma cells. Biochem Pharmacol 2015; 97: 38-50.

12. World Cancer Report, World Health Organization, 2014.

13. Bast RC, Hennessy B, Mills GB. The biology of ovarian cancer: new opportunities for translation. Nature Rev Cancer 2009; 9: 415-28.

14. Azuma M, Tamatani T, Ashida Y, Takashima R, Harada K, Sato M. Cisplatin induces apoptosis in oral squamous carcinoma cells by the mitochondria-mediated but not the NF-kappaB-suppressed pathway. Oral Oncol 2003; 39: 282-9.

15. Córdova I, León LG, León F, San Andrés L, Luis JG, Padrón JM. Synthesis and antiproliferative activity of novel sugiol beta-amino alcohol analogs. Eur J Med Chem 2006; 41: 1327-32.

16. Tu WC, Wang SY, Chien SC, et al. Diterpenes from Cryptomeria japonica inhibit androgen receptor transcriptional activity in prostate cancer cells. Planta Med 2007; 73: 1407-9.

17. Siddiqui S, Siddiqui BS, Faizi S, Mahmood T. Tetracyclic triterpenoids and their derivatives from Azadirachta indica. J Nat Prod 1988; 51: 30-43.

18. Ferreira CG, Epping M, Kruyt FA, Giaccone G. Apoptosis. Clin Cancer Res 2002; 8: 2024-34.

19. Brown JM, Wouters BG. Apoptosis, p53, and tumor cell sensitivity to anticancer agents. Cancer Res 1999; 59: 1391-9.

20. Waldman T, Zhang Y, Dillehay L, et al. Cell-cycle arrest versus cell death in cancer therapy. Nat Med 1997; 3: 1034-6.

21. Feng W, Li L, Xu X, Jiao Y, Du W. Up-regulation of the long non-coding RNA RMRP contributes to glioma progression and promotes glioma cell proliferation and invasion. Arch Med Sci 2017; 13: 1315-21.

22. Takano S, Gately S, Neville ME, et al. Suramin, an anticancer and angiosuppressive agent, inhibits endothelial cell binding of basic fibroblast growth factor, migration, proliferation, and induction of urokinase-type plasminogen activator. Cancer Res 1994; 54: 2654-60.

23. Roberts PJ, Der CJ. Targeting the Raf-MEK-ERK mitogen-activated protein kinase cascade for the treatment of cancer. Oncogene 2007; 26: 3291-310. 